\title{
Nase frei dank Meersalz
}

— Gerade zur Herbstzeit leiden viele Patienten unter Erkältung und Schnupfen. Zu den Symptomen zählen meist eine stark erschwerte Nasenatmung, Niesreiz und eine gerötete und geschwollene $\mathrm{Na}$ senschleimhaut. Hier wirkt sich die hypertone Meersalzlösung von Rinupret ${ }^{\circledR}$ Pflege Nasenspray (2,7\% statt üblicherweise 0,9 $\%)$ besonders günstig auf die Atemwege aus. Denn sie entzieht der geschwollenen Nasenschleimhaut das überschüssige Wasser. Dieser „osmotische Effekt" führt dazu, dass die Schleimhaut abschwillt, sich die Durchgängigkeit der oberen Atemwege verbessert und man wieder freier atmen kann. Aloe vera wirkt darüber hinaus wundheilungsfördernd und befeuchtend, während Eukalyptusöl belebt und erfrischt. So werden Nasenschleimhäute vor Austrocknung und weiteren Belastungen geschützt.

Sollte sich einmal ein Schnupfen ankündigen, empfiehlt es sich, den Symptomen frühzeitig mit einem natürlichen, schleimlösenden und entzündungshemmenden Arzneimittel wie etwa Sinupret ${ }^{\circledR}$ zu begegnen. Das lindert nicht nur die Beschwerden, sondern verringert zudem die Gefahr einer Entzündung der Nasennebenhöhlen.

Rinupret ${ }^{\circledR}$ Pflege Nasenspray eignet sich besonders für die Begleitbehandlung von entzündeter Nasenschleimhaut, verstopfter

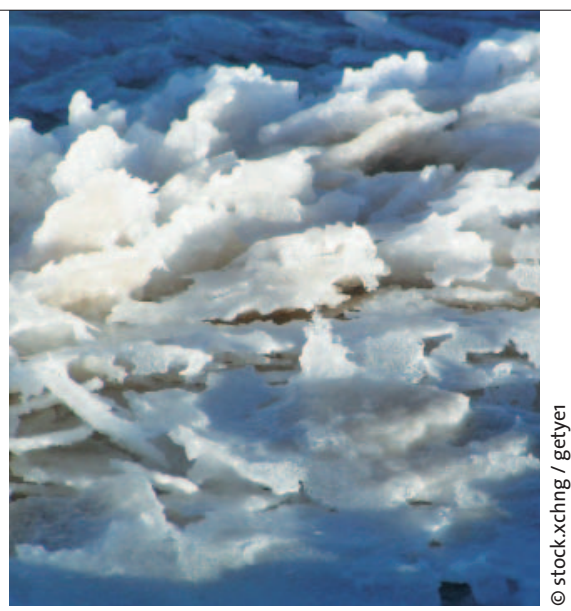

Nase und Schnupfen und ist in Originalpackungen mit $15 \mathrm{ml}$ Lösung erhältlich.

Nach Informationen von Bionorica, Neumarkt

\section{SCIT: Adjuvans verkürzt Aufdosierung und reduziert Wirkstoffmenge}

- Mit AVANZ $^{\circledR}$ steht ab sofort eine hypoallergene, immunologisch verstärkte subkutane Immuntherapie (SCIT) zur Verfügung. Möglich wird dies durch ein optimiertes Allergen-Aluminiumhydroxid-Verhältnis. Neben der Depotwirkung bietet das Adjuvans Aluminiumhydroxid einen verstärkenden Effekt auf die immunologische Wirkung der spezifischen Immuntherapie. Wie stark dieser Effekt ist, zeigt das Ergebnis einer Untersuchung. Analysiert wurde dabei die Immunantwort bei der 1. bis 5 . Immunisie- rung (nach Aufdosierung) von ALK-depot SO ${ }^{\circledR} 100.000 \mathrm{SG} / \mathrm{ml}$ und die von AVANZ $^{\circledR}$ 15.000 SQ+/ml. Bei Letzterem wurde das Allergen-Aluminiumhydroxid-Verhältnis auf 1:3 optimiert. Gezeigt werden konnte, dass AVANZ $^{\circledR}$ mit diesem optimierten Verhältnis eine ähnlich hohe Antikörperproduktion wie ALK-depot SO ${ }^{\circledR}$ bewirkt.

Bestätigt wird die effiziente immunologische Wirkung von AVANZ ${ }^{\circledR}$ auch durch Ergebnisse einer aktuellen klinischen Studie mit 470 Patienten. Die Untersuchung zeigte nach fünf Injektionen Aufdosierung und zwei Injektionen Erhaltungsdosis mit AVANZ $^{\circledR}$ einen signifikanten Anstieg des IgG4 und des IgE-blockierenden Faktors im Vergleich zum Ausgangswert. Aufgrund der schnellen Aufdosierung in nur fünf Schritten sowie einem Dosisintervall von $6 \pm 2$ Wochen in der Erhaltungstherapie ist die Therapie mit AVANZ $^{\circledR}$ für die Patienten sehr angenehm.

Nach Informationen von ALK-Abelló, Wedel

\section{Hyaluronsäurehaltige Halstabletten lindern Halsschmerzen}

— Hyaluronsäure ist seit Jahren in der Orthopädie, Dermatologie oder Ophthalmologie etabliert. Nun wird das Polysaccharid auch bei Erkrankungen der oberen Atemwege eingesetzt und „verspricht einen deutlichen Einfluss auf die Krankheitsbilder akute und chronische Pharyngitis", wie Prof. Hans Behrbohm, Berlin, erklärte. GeloRevoice ${ }^{\circledR}$ Halstabletten enthalten als wesentlichen Bestandteil neben Carbomer und Xanthan Natriumhyaluronat, das in der Lage ist, große Wassermengen zu binden. Beim Lutschen der Halstabletten entsteht ein Hydrogelkomplex, der sich gleichmäßig als Schutzfilm auf die Mund- und Rachenschleimhaut verteilt. Weil Hyaluronsäure darüber hinaus die Wundheilung fördert, werden gereizte Bereiche geschützt und können regenerieren. In der Folge lindert die mucilaginöse Behandlung Beschwerden wie Heiserkeit, Hustenreiz, Halskratzen, trockene Schleimhaut und resultierende Schmerzen.

Die Effektivität der Halstabletten demonstriert eine prospektive multizentrische Anwendungsbeobachtung von 2008. $278 \mathrm{~Pa}$ tienten mit den Symptomen einer Erkrankung der oberen Atemwege, erhielten GeloRevoice ${ }^{\circledR}$ Halstabletten. Unter dreitägiger Anwendung reduzierte sich die Intensität der Symptome Heiserkeit, Halsschmerzen und Kratzen im Hals um im Mittel $60 \%$.
Nach Bedarf sollen bis zu sechs Halstabletten über den Tag verteilt langsam gelutscht werden, empfiehlt der Hersteller. Bei Kindern unter sechs Jahren sowie Patienten mit Überempfindlichkeit gegen Bestandteile des Medizinprodukts sollen sie nicht angewendet werden. Das gleiche gilt für Patienten mit Phenylketonurie oder stark eingeschränkter Speichelproduktion, etwa dem Sjögren-Syndrom.

koc

Pressekonferenz „Neu: GeloRevoice ${ }^{\circledR}$ - effektiver Schutz der Mund- und Rachenschleimhaut", Düsseldorf, 25. September 2009 Veranstalter: Pohl-Boskamp, Hohenlockstedt 